\title{
Eine Antibiotikaprophylaxe kann das Pneumonierisiko nicht reduzieren
}

Fragestellung: Kann eine Pneumonie nach Schlaganfall bei Patienten mit Schluckstörungen durch die prophylaktische Gabe von Antibiotika reduziert werden?

Hintergrund: Etwa $10 \%$ aller Patienten mit einem akuten Schlaganfall entwickeln auf der Stroke Unit eine Pneumonie. Infektiöse Erkrankungen, wie Pneumonien verschlechtern die Prognose des Schlaganfalls. Bei bis zu 50\% der Patienten mit Schlaganfall fällt in der Akutphase eine Schluckstörung auf. Diese Patienten haben ein zwei- bis dreifach erhöhtes Risiko für die Entwicklung einer Pneumonie.

Im Moment werden Patienten, die eine Pneumonie entwickeln, antibiotisch behandelt. Bisher wurde nicht untersucht, ob bei dieser Patientenpopulation eine prophylaktische antibiotische Behandlung die Entwicklung von Pneumonien verhindert und die Prognose der Patienten verbessert.

Patienten und Methodik: Es handelt sich um eine prospektive multizentrische clusterrandomisierte offene Studie mit einer verblindeten Beurteilung der Endpunkte, die in 48 Stroke Units in Großbritannien durchgeführt wurde. Ausgeschlossen wurden Patienten, bei denen Kontraindikationen für eine Gabe von

Kalra L, Irshad S, Hodsoll J et al; STROKE-INF Investigators. Prophylactic antibiotics after acute stroke for reducing pneumonia in patients with dysphagia (STROKE-INF): a prospective, cluster-randomised, open-label, masked endpoint, controlled clinical trial. Lancet 2015; DOI: http://dx.doi.org/10.1016/S01406736(15)00126-9
Antibiotika bestanden, Patienten mit vorbestehender Schluckstörung oder bekannten Infektionen und $\mathrm{Pa}$ tienten mit schlechter Prognose.

Die einzelnen Schlaganfallzentren wurden in zwei Gruppen randomisiert. Die Antibiotikagruppe behandelte die Patienten entweder über sieben Tage mit Amoxi- cillin in Kombination mit Clarithromycin, die Kontrollgruppe verwendete ausschließlich ihre Standardtherapie. Die antibiotische Behandlung musste innerhalb von 48 Stunden nach Beginn der klinischen Symptomatik eingesetzt werden. Alle Patienten mussten in der Schluckdiagnostik eine Dysphagie aufweisen. Der primäre Outcome war die Entwicklung einer Pneumonie in den ersten 14 Tagen.

Ergebnisse: Zwischen April 2008 und Mai 2014 wurden 24 Stroke Units in die Antibiotikagruppe und 24 Stroke Units in die Standardtherapiegruppe randomisiert. In der Studie befanden sich insgesamt 1.224 Patienten. Für die Endauswertung standen 615 Patienten in der Antibiotika- und 602 in der Kontrollgruppe zur Verfügung.

Die Patienten waren im Mittel 78 Jahre alt (55\% Frauen), $90 \%$ hatten eine Ischämie und $10 \%$ eine Blutung. Bei $30 \%$ der Patienten wurde eine systemische Thrombolyse durchgeführt. Bei $25 \%$ musste das Antibiotikum über eine Nasensonde appliziert werden. Der mittlere Wert auf der NIHSS-Skala lag bei 14. Die Inzidenz einer Pneumonie betrug 71 von 564 Patienten in der Antibiotikagruppe (13\%) versus 52 von 524 Patienten in der Kontrollgruppe (10\%).

Die Odds Ratio betrug 1,12 und war statistisch nicht signifikant. In der Studie fiel auf, dass in der Antibiotikagruppe weniger Atemwegsinfekte auftraten. Bei den klinischen Parametern ergab sich kein Unterschied in der Schwere des Schlaganfalls gemessen mit den NIHSS-Score nach 14 Tagen, in der Sterblichkeit nach 14 und 90 Tagen sowie im funktionellen Outcome gemessen mit der modifizierten Rankin Skala. Dasselbe galt für die Lebensqualität.

Schlussfolgerungen: Bei Schlaganfallpatienten mit Schluckstörungen kann eine prophylaktische antibiotische Behandlung die Entwicklung einer Pneumonie nicht verhindern.

\section{Vordringlich ist, Aspirationen zu verhindern}

Es gibt in letzter Zeit zwei große und gut durchgeführte Studien zum präventiven Einsatz von Antibiotika bei Patienten mit Schlaganfall. Die Preventive Antibiotics in Stroke Study (PASS), die in Holland durchgeführt wurde, zeigte bei einer prophylaktischen Antibiotikabehandlung bei allen Schlaganfallpatienten zwar eine reduzierte Rate an Atemwegsinfektionen, aber keine Konsequenz für den funktionellen Outcome [1]. Die hier vorliegende englische Studie ist sehr viel praxisrelevanter, da sie untersucht, ob bei Patienten mit Schluckstörungen, bei denen per se das Pneumonierisiko deutlich erhöht ist, eine prophylaktische antibiotische Behandlung die Ent- wicklung von Pneumonien verhindert und die Prognose verbessert. Beides war nicht der Fall. Auf Stroke Units kann daher beruhigt abgewartet werden, ob sich eine Pneumonie entwickelt und in diesem Fall dann rasch antibiotisch behandelt werden. Am wichtigsten ist es natürlich, durch eine frühe Diagnose und eine entsprechende Therapie Aspirationen und damit Pneumonien per se zu verhindern.

\footnotetext{
Referenz:

1. Westendorp WF et al. Lancet. 2015; 385: 1519-26
} 